\title{
Comparação da análise da frente de onda e da sensibilidade ao contraste em olhos pseudofácicos com implante de lentes intra-oculares esférica e asférica
}

\author{
Wavefront analysis and contrast sensitivity comparison \\ between spheric and aspheric intraocular lenses
}

Wilson Takashi Hida', Íris de Souza Yamane', Antonio Francisco Pimenta Motta', Marcel Tadeu Silva', Emerson Alves', Newton Kara José Junior', Celso Takashi Nakano'

\section{RESUMO}

Objetivo: Comparar a análise da frente de onda e a sensibilidade de contraste pósoperatórias em pacientes submetidos à facoemulsificação com implante de lentes intraoculares esféricas num olho e asféricas no olho contralateral.Métodos: Este estudo prospectivo comparativo, randomizado, duplo-cego, incluiu 32 olhos de 16 pacientes. Uma avaliação oftalmológica completa foi realizada durante as visitas antes e depois da cirurgia (no primeiro, terceiro, sétimo, e nonagésimo dias pós-cirúrgicos), que incluiu acuidade visual, teste de sensibilidade ao contraste, aberrometria. Todos exames (menos aberrometr-=ia) foram feitos monocularmente com pupilas não dilatadas. Os pacientes foram submetidos a facectomia ultra-sônica convencional com implante de uma lente asférica SN60WF num olho e esférica SN60ATno outro. Resultados: A acuidade visual média com a melhor correção foi -0,146 LogMAR no grupo da lente intra-ocular esférica, e -0.165 logMAR no grupo da lente intra-ocular asférica sob condições fotópicas, $-0,003$ LogMAR sob condições mesópicas. A sensibilidade ao contraste foi semelhante em ambos os grupos tanto em condições fotópicas e mesópicas. Em relação às aberrações ópticas ocorreu uma diferença significativa nas aberrações totais $(1,09 \pm 0,56$ no grupo asférico e 1,52 \pm 1,19 no grupo esférico) e no componente esférico das aberrações de alta ordem $(0,422 \pm 0,202$ no grupo asférico e $0,124 \pm 0,087$ no grupo esférico). Conclusão: As duas lentes intra-oculares promoveram uma excelente visão em situações de alto contraste, e os olhos implantados com a lente asférica tiveram menos aberrações totais e esféricas que aqueles implantados com a lente esférica.

Descritores:Lentes intra-oculares; Facoemulsificação;Sensibilidades de contraste; Visão

\footnotetext{
${ }^{1}$ Fellow do Setor da Catarata da Faculdade de Medicina da Universidade de São Paulo - USP - São Paulo (SP), Brasil; ${ }^{2}$ Assistente do Setor de Catarata do Departamento de Oftalmologia da Faculdade de Medicina da Universidade de São Paulo - USP - São Paulo (SP), Brasil.

Departamento de Oftalmologia da Faculdade de Medicina da Universidade de São Paulo - USP - São Paulo (SP), Brasil
} 


\section{INTRODUÇÃO}

$\mathbf{N}$ ovos aparelhos e tecnologias vêm sendo desenvolvidos para medir os defeitos ópticos, como os refratores automatizados e analisadores de frente de onda. Estes últimos podem medir as aberrações do olho tanto de baixa ordem, quanto aquelas de altas ordens, por meio da aberrometria de Hartmann-Shack, Tscherning, Tracey ou esquiascopia espacial dinâmica(OPD-Scan) ${ }^{(1-3)}$

Com o aumento da idade ocorre concomitantemente um aumento das aberrações de alta ordem ${ }^{4}$ o que leva a uma diminuição na qualidade da visão pela diminuição da sensibilidade ao contraste que consiste na habilidade de distinguir os detalhes das imagens dependendo da luminosidade ambiente ${ }^{(5.9)}$. Essa sensibilidade pode ser medida por meio de testes variados e todas estas alterações são exacerbadas como o surgimento da catarata.

$\mathrm{Na}$ tentativa de promover benefícios adicionais na qualidade visual do paciente pseudofácico, as lentes intra-oculares (LIOs) ${ }^{(10)}$ foram aperfeiçoadas por meio de um desenho asférico, de modo a corrigir as aberrações esféricas positivas da córnea ${ }^{(11-16)}$. Com esse aperfeiçoamento foi desenvolvida uma lente asférica a SN60WF, desenvolvida a partir da lente SN60AT, ambas produzidas pelos Laboratórios Alcon (Fort Worth, Texas, USA). A primeira oferece uma redução nas aberrações esféricas com uma visão melhor no período após a cirurgia do que o segundo modelo ${ }^{(9,11,14)}$.

O objetivo deste estudo é comparar a acuidade visual final, análise da frente de onda e a sensibilidade de contraste pós-operatórias em pacientes submetidos à facoemulsificação com implante de lentes intra-oculares esféricas num olho e asféricas no olho contra-lateral.

Atentamos para a metodologia deste trabalho associada à análise aberrométrica com OPD-scan (Nidek, Tóquio, Japão), na qual não foi encontrado na revisão da literatura. O estudo apresenta uma metodologia criteriosa imprescindível para avaliação das diferentes LIOS.

\section{Métodos}

Este estudo prospectivo,comparativo,randomizado, duplo-cego incluiu 32 olhos de 16 pacientes selecionados entre fevereiro e junho de 2006. Os critérios de inclusão foram: idade entre 45 e 70 anos; presença de catarata bilateral; astigmatismo corneal menor que 1,00 dioptria em ambos os olhos; diâmetro pupilar mínimo de 3,5 $\mathrm{mm}$ em condições mesópicas, medido por pupilômetro de Colvard
(Oásis Medical, Glendora, Califórnia, EUA); aberrações corneais semelhantes nos dois olhos; ausência de cirurgias oculares prévias, de uso de medicações hipotensoras, ou de outras doenças oculares quaisquer que poderiam afetar o desempenho da visão pós-operatória pela diminuição da sensibilidade de contraste, como retinopatia causada pela diabetes mellitus ${ }^{(17)}$. Aqueles pacientes que preenchiam os critérios de inclusão foram informados sobre a necessidade de exames complementares adicionais como medida das aberrações ópticas e da sensibilidade de contraste e somente foram incluídos aqueles que concordaram com a realização dos mesmos. Nenhum paciente foi excluído devido a complicações no ato cirúrgico ou no período pós-operatório, por dúvidas quanto ao implante da LIO dentro do saco capsular, ou descentração da mesma maior que $0,5 \mathrm{~mm}$ avaliados pelo exame na lâmpada de fenda.

Uma avaliação oftalmológica completa foi realizada durante todas as visitas pré e pós-operatórias (no primeiro, terceiro, sétimo, e nonagésimo dias pós-cirúrgicos) e incluiu medidas das acuidades visuais com e sem correção, teste de sensibilidade ao contraste, tonometria, biomicroscopia, fundoscopia e aberrometria. A técnica cirúrgica consistiu na realização da facectomia ultra-sônica convencional com implante de uma lente asférica (Acrysof ${ }^{\circledast} \mathrm{IQ}^{\circledast}$ - SN60WF) num olho e esférica (Acrysof ${ }^{\oplus}$ Natural $^{\oplus}$ - SN60AT) no outro.

Os pacientes selecionados tiveram poder dióptrico da LIO calculado pela técnica ultra-sônica de imersão ${ }^{(18)}$, realizada por um único examinador experiente (A.F.P.M.), usando o biômetro Ocuscan RXP ${ }^{\oplus}$ (Laboratórios Alcon, Fort Worth, Texas, USA), e as fórmulas usadas para selecionar o poder da LIO foram a Hoffer-Q (quando o comprimento axial foi menor que $22 \mathrm{~mm}$ ) e SRK/T (quando o comprimento axial foi maior que $22 \mathrm{~mm}$ ), com refração alvo pós-operatória plana ou a mais próxima.

As cirurgias foram feitas por único e experiente cirurgião (C.T.N.), no centro cirúrgico do HC-FMUSP,com técnica cirúrgica padronizada que consistiu em anestesia tópica, incisão em córnea clara, auto-selante, de 2,75 mm no meridiano corneal mais curvo, capsulorrexe circular contínua, hidrodissecção com lidocaína a 1\% sem preservativos diluída em $10 \mathrm{ml}$ de solução salina balanceada. Em seguida, uso de viscoelásticos dispersivo Celoftal $^{\varpi}$ (Hidroxipropil metilcelulose) e coesivo Provisc ${ }^{\oplus}$ (Hialuronato de Sódio a $1 \%$ ) produzidos pelos Laboratórios Alcon (Fort Worth, Texas, USA) pela técnica de "soft-shell" "(19) ,facoemulsificação convencional usando o sistema Infiniti'(Laboratórios Alcon, Fort Worth, Texas, USA) e implante da LIO dentro do saco capsular 
com uso de injetor Royale ${ }^{\varpi}$ Asico, Westmont, Illinois, EUA). No pós-operatório foram utilizados topicamente: quinolona de quarta geração (moxifloxacino 0,5\%, Vigamox ${ }^{\varpi}$,Laboratórios Alcon, Fort Worth, Texas, USA) 01 gota de 6 em 6 horas por 07 dias, associada a corticóide tópico (dexametasona 1\%,Maxidex ${ }^{\circledast}$,Laboratórios Alcon ,Fort Worth, Texas, USA) inicialmente 01 gota de $4 \mathrm{em} 4$ horas, que foi regredido progressivamente ao longo de trinta dias.

Os exames foram realizados monocularmente, com pupilas não dilatadas, sem e com a melhor correção óptica. A acuidade visual foi medida por meio da tabela do "Early Treatment Diabetic Retinopathy Study" (ETDRS) ${ }^{(20)}$ chart (No. 2106, Precision Vision, Aurora, Colorado, USA), com contrastes de $100 \%$ e $9 \%$ colocados há uma distância de quatro metros sob condições controladas de luminosidade fotópicas estabelecidas em $85 \mathrm{~cd} / \mathrm{m}^{2}$, de modo a avaliar a acuidade visual fotópica em diferentes condições de contraste.

A sensibilidade de contraste também foi medida sob condições fotópicase posteriormente em condições mesópicas $\left(6 \mathrm{~cd} / \mathrm{m}^{2}\right)$ com o VCTS ${ }^{\varpi} 6000$ (Vistech consultants, Dayton, Ohio, EUA). Esta tabela apresenta linhas em cinco freqüências espaciais entre 1,5 e 18 ciclos/grau (cpd). A base logarítmica 10 dos valores obtidos foram realizadas para se obter os valores de sensibilidade de contraste que foram usados na análise estatística.

Por meio do pupilômetro infra-vermelho de Colvard (Oásis Medical, Glendora, Califórnia, EUA) os diâmetros pupilares de todos os olhos foram medidos sob as mesmas condições de iluminação, após dilatação farmacológica com tropicamida a 1\% (Mydryacyl ${ }^{\varpi}$, Laboratórios Alcon, Fort Worth, Texas, USA,), sendo então encaminhados para exame de aberrometria no mínimo $6 \mathrm{~mm}$ de diâmetro ${ }^{(21)}$. As aberrações ópticas foram medidas pelo OPD Scan ${ }^{\oplus}$ (Nidek, Tóquio, Japão) .

A significância foi testada ao nível de $5 \%$ ou 0,05 por meio dos testes de Kruskal-Wallis, Mann-Whitney e Qui-quadrado (bicaudal), ajustando-se o nível de significância quando necessário para 1,7\% .

\section{Resultados}

Dezesseis pacientes foram incluídos no estudo num total de 32 olhos, e receberam lente asférica num olho e esférica no outro. Não houveram complicações intra-operatórias, ou no período de acompanhamento após a cirurgia. A média da idade foi de $65,13 \pm 7,34$ anos.

Na última visita pós-operatória, a cápsula posterior foi verificada em todos os olhos, não sendo verificada presença de opacificação da mesma.
As acuidades visuais médias com a melhor correção, medidas pelas tabelas com contraste de $100 \%$ (grupo esférica $-0,24$ a 0,28 ; grupo asférica $-0,3$ a 0,1 ) e $9 \%$ (grupo esférica -0,1 a 0,3; grupo asférica -0,3 a 0,22) estão na tabela 1 . Os olhos com lentes asféricas apresentaram melhores níveis de acuidade visual corrigida no contraste de $9 \%$ em comparação às lentes esféricas, mas esta diferença não foi estatisticamente significante.

Os resultados da sensibilidade de contraste em condições fotópicas são mostrados no gráfico 1, e em condições mesópicas no gráfico 2 . Não observamos diferença estatisticamente significativa em condições fotópicas nem mesópicas, em nenhuma das freqüências espaciais analisadas. Não foi encontrada nenhuma diferença estatisticamente significante em relação ao limite mínimo do intervalo de normalidade estabelecido pela tabela VCTS ${ }^{\circledast} 6000$ (Vistech consultants, Dayton, Ohio, EUA).

As aberrações ópticas foram analisadas em todas as suas variáveis (da quarta a oitava ordem). Os valores das aberrações são mostrados na figura 3. Diferenças com significâncias estatísticas foram observadas nas aberrações totais e nas aberrações esféricas. A média das aberrações totais foi de $1,09( \pm 0,56)$ no grupo asférico e de $1.52( \pm 1,19)$ no grupo esférico $(\mathrm{p}=0,0047)$.O mesmo comportamento foi observado para o componente esférico das aberrações oculares de alta ordem, com media de $0,422( \pm 0,202)$ no grupo esférico e 0,124 $( \pm 0,087)$ no grupo asférico $(\mathrm{p}=0,0046)$.Observa-se ainda uma menor incidência de aberrações em todos outros componentes avaliados nos olhos implantados com a lente asférica, comparando-as com aqueles implantados com a esférica, porém sem significância estatística.

\section{Discussão}

Novas lentes intra-oculares, técnicas operatórias e exames permitem um melhor manejo do paciente com boa visão final pós-operatória em diversas situações cotidianas com diferentes condições de luminosidade e contraste.

A acuidade visual em condições de alto contraste (tabela do ETDRS a 100\% de contraste) e em baixo contraste (tabela do ETDRS a 9\% de contraste) testadas para as lentes intra-oculares avaliadas nesse estudo tiveram resultados similares sem diferenças estatísticas, com uma tendência para melhor desempenho para a lente asférica em ambas situações (Tabela 1).

Diversas inovações nas lentes intra-oculares foram desenvolvidas no intuito de satisfazer essas expectativas com uma boa visão funcional e os cirurgiões de 
Tabela 1

Acuidade visual para distância com a melhor correção na tabela ETDRS com contraste de 100\% e $9 \%$ (em LogMAR)

\begin{tabular}{lccc}
\hline & SN60AT & SN60WF & Kruskal-Wallis test \\
\hline ETDRS 100\% & $\mathrm{N}=16$ olhos & $\mathrm{N}=16$ olhos & \\
Média $( \pm$ DP $)$ & $-0,146( \pm 0,125)$ & $-0,165( \pm 0,128)$ & $\mathrm{KW}=1,61$ \\
ETDRS 9\% & & & $\mathrm{P}=0,45$ \\
Média $( \pm$ DP $)$ & $-0,024( \pm 0,056)$ & $-0,003( \pm 0,176)$ & $\mathrm{KW}=0,14$ \\
\end{tabular}

Acuidade visual expressa em LogMar DP: Desvio Padrão

Gráfico 1

\section{Comparação da sensibilidade de contraste em condições fotópicas entre a lente intra-ocular esférica e asférica $(n=32)$}

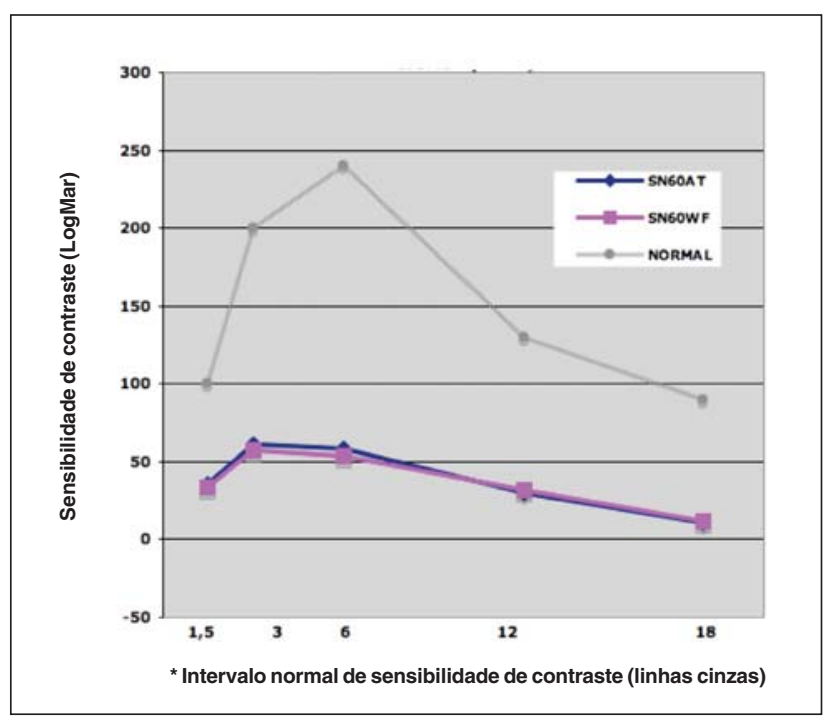

catarata têm várias opções para escolher ${ }^{(9,15-16,622-25)}$.Todas as lentes implantadas nos pacientes desse estudo tinham pigmento amarelo com propriedades de filtro para o espectro azul da luz visível, o que poderia interferir nos exames realizados. Porém, vários trabalhos realizados mostram que não existe interferência na sensibilidade de contraste, acuidade visual, em condições fotópicas e mesópicas com alta luminosidade, com ou sem glare, ou mesmo na distinção das cores ${ }^{(7,26-27)}$.

A diminuição na sensibilidade de contraste que ocorre com o aumento da idade ${ }^{(4)}$ leva a uma perda na qualidade da visão, que é mais prejudicada ainda com o desenvolvimento de catarata. Essa capacidade do sistema óptico de diferenciar os objetos de tonalidades e cores

\section{Gráfico 2}

\section{Comparação da sensibilidade de contraste em condições mesópicas entre a lente intra-ocular esférica e asférica $(n=32)$}

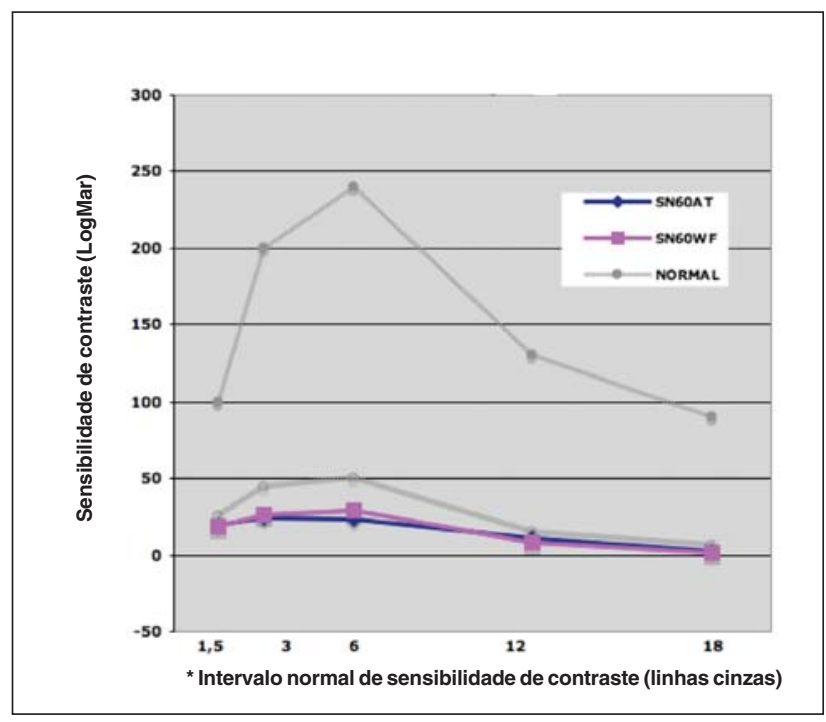

similares no dia a dia ganhou maior importância com o melhor entendimento das aberrações oculares, pois reflete melhor a visão funcional e se correlaciona com o desempenho da visão ${ }^{(2)}$ e a qualidade de vida ${ }^{(28)}$. Bellucci et al. observaram melhor desempenho de uma lente intraocular asférica, comparada com uma esférica ${ }^{(9)}$, porém outro estudo mostra um melhor desempenho na função de modulação de transferência monocromática, mas não observada na policromática, comparando uma lente asférica com uma esférica convencional ${ }^{(29)}$. Muñoz em um estudo recentemente publicado, não observou diferenças significantes comparando uma lente asférica com outras duas outras lentes esféricas ${ }^{(16)}$, concordando com os resultados do presente estudo onde não observamos diferença 


\section{Gráfico 3}

Comparação da análise de frente de onda entre a lente intra-ocular esférica e asférica $(n=32)$

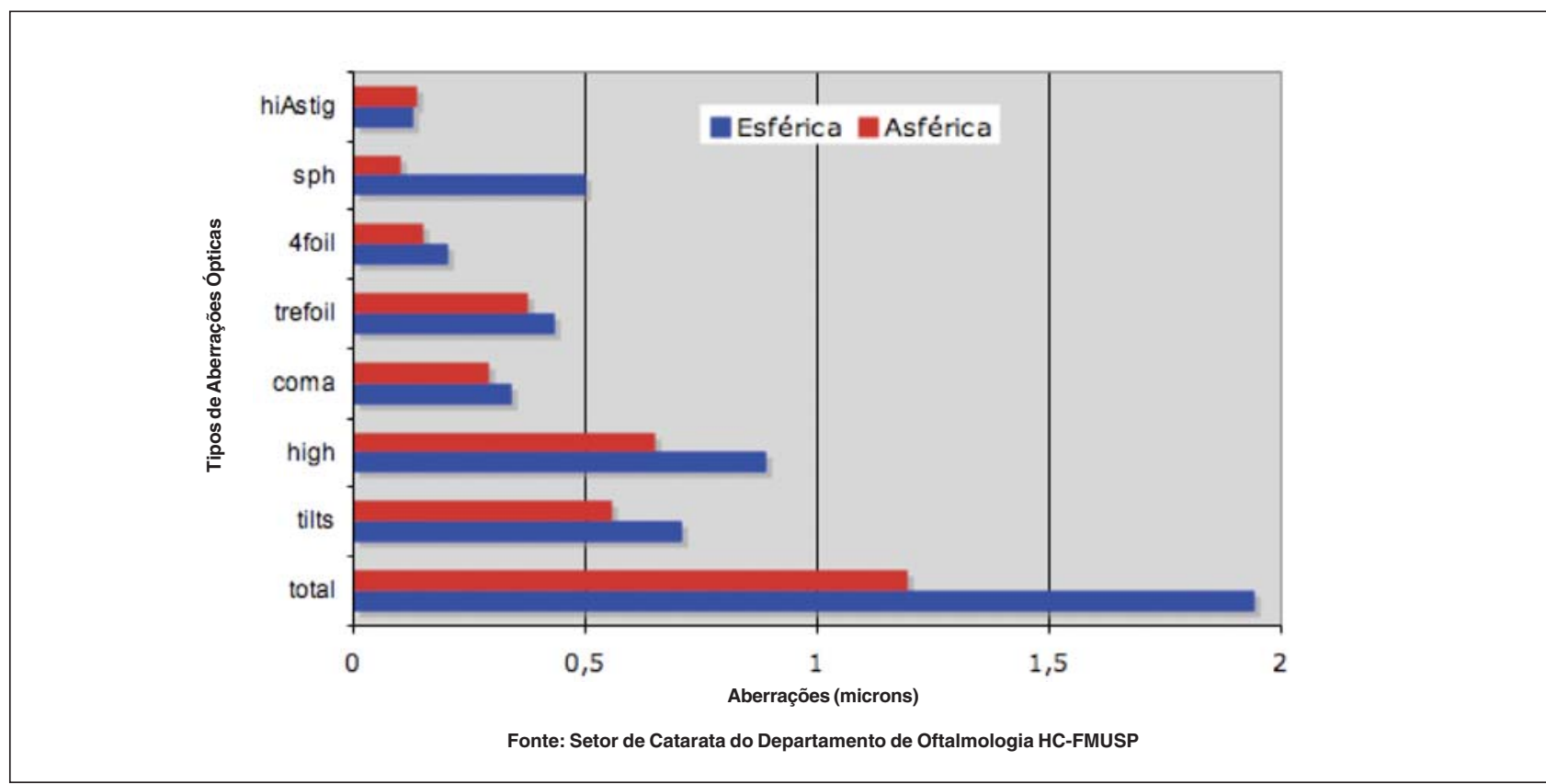

estatisticamente significante entre as lentes estudadas tanto em condições fotópicas, quanto mesópicas.

Com relação às aberrações ópticas, elas estão aumentadas no paciente idoso conseqüente ao aumento do diâmetro ântero-posterior do cristalino e perda da capacidade de compensar as aberrações esféricas positivas corneanas. As novas lentes intra-oculares asféricas se propõem a diminuir as aberrações de alta ordem, incluindo o componente esférico ${ }^{(14)}$. No nosso estudo ocorreu diferença estatística significante entre as lentes estudadas, quando se analisou as aberrações totais $(\mathrm{p}=0,0047)$ e o mesmo foi observado com relação às aberrações esféricas $(\mathrm{p}=0,0046)$. Resultados semelhantes foram encontrados num estudo experimental onde as lentes esféricas tiveram mais aberrações esféricas quando comparadas às lentes asféricas ${ }^{(5)}$,e Padmanabhan et al. observaram mais aberrações esféricas comparando olhos pseudofácicos com olho fácicos de jovens míopes, concluindo ser uma consequiência do design da lente ${ }^{(30)}$, pois em outro estudo o mesmo autor observa diminuição nas aberrações esféricas com uma lente com design modificado, mantendo porém um mesmo RMS "Root Mean Square" comparada a duas outras lentes monofocais avaliadas ${ }^{(31)}$, concordando com Rohart et al. ${ }^{(32)}$.

Em conclusão, as duas lentes intra-oculares promoveram uma excelente visão em situações de alto con- traste, e os olhos implantados com a lente asférica tiveram menos aberrações totais e esféricas que aqueles implantados com a lente esférica.

\section{Abstract}

Purpose: Compare the wavefront and contrast sensitivity of bilateral pseudophakic patients with aspheric in one eye and spheric intraocular lenses in the other. Methods: This prospective, comparative, randomized study, double masked, included 32 eyes of 16 patients. A complete ophthalmic evaluation was done before, and after surgery at days 1,3, 7 and 90, and included best corrected visual acuity, the contrast sensitivity, under photopic and mesopic conditions, and aberrometry. Almost all exams were done monocularly with undilated pupils (aberrometry under pharmacological dilatation). The patients eyes were operated with conventional phacoemulsification, with aspheric intraocular lenses in one eye, and spherical in the other. Results: The visual acuity with the best correction was -0.146 in the spheric group, and -0.165 $\log M A R$ in the aspheric group in photopic conditions, and 0.024 and 0.003 LogMAR under mesopic conditions. The contrast sensitivity under photopic and mesopic conditions was similar comparing the two groups. In regard to optical aberrations there was a significant diference between the 
total $(1.09 \pm 0.56$ in the aspheric, and $1.52 \pm 1.19$ in the spherical group) and spherical component of high order aberrations $(0.422 \pm 0.202$ in spheric group and $0.124 \pm$ 0.087 in the aspheric group).Conclusion:Both groups reached excellent postoperative visual acuity in high contrast situations, however aspheric intraocular lenses group had lower total and spherical aberrations, comparing to the spheric intraocular lenses group.

Keywords: Lenses, intraocular; Phacoemulsification; Contrast sensitivity; Vision

\section{ReferênCias}

1. Guirao A, Redondo M, Geraghty E, Piers P, Norrby S, Artal P. Corneal optical aberrations and retinal image quality in patients in whom monofocal intraocular lenses were implanted. Arch Ophthalmol. 2002;120(9):1143-51.

2. Packer M, Fine IH, Hoffman RS. Wavefront technology in cataract surgery. Curr Opin Ophthalmol. 2004;15(1):56-60.

3. Packer M, Fine IH, Hoffman RS, Piers P. Aberrations after intraocular lens implantation. J Cataract Refract Surg. 2006;32(2):184-5; author reply 185-6.Comment on: J Cataract Refract Surg. 2005; 31(4):725-34.

4. Alió JL, Schimchak P, Negri HP, Montés-Micó R. Crystalline lens optical dysfunction through aging. Ophthalmology. 2005;112(11):2022-9.Comment in: Ophthalmology. 2007; 114(3):618; author reply 619.

5. Marcos S, Barbero S, Jiménez-Alfaro I. Optical quality and depth-of-field of eyes implanted with spherical and aspheric intraocular lenses. J Refract Surg. 2005;21(3):223-35.

6. Friström B, Lundh BL. Colour contrast sensitivity with different intraocular lens materials in the right and left eyes in same day surgery. Acta Ophthalmol Scand. 2005;83(4):443-7.

7. Rodríguez-Galietero A, Montés-Micó R, Muñoz G, AlbarránDiego C. Comparison of contrast sensitivity and color discrimination after clear and yellow intraocular lens implantation. J Cataract Refract Surg. 2005;31(9):1736-40.

8. Oliveira F, Muccioli C, Silva LMP, Soriano ES, Souza CEB, Belfort Jr R.Avaliação da sensibilidade ao contraste e da estereopsia em pacientes com lente intra-ocular multifocal. Arq Bras Oftalmol. 2005;68(4):439-43.

9. Bellucci R, Scialdone A, Buratto L,Morselli S, Chierego C, Criscuoli A, et al. Visual acuity and contrast sensitivity comparison between Tecnis and AcrySof SA60AT intraocular lenses: A multicenter randomized study. J Cataract Refract Surg. 2005;31(4):7127.Erratum in: J Cataract Refract Surg. 2005; 31(10):1857.

10. Ridley H. Intra-ocular acrylic lenses after cataract extraction. Lancet. 1952;1(6699):118-21.

11. Altmann GE. Wavefront-customized intraocular lenses. Curr Opin Ophthalmol. 2004;15(4):358-64.

12. Christie B, Nordan L, Chipman R, Gupta A. Optical performance of an aspheric multifocal intraocular lens. J Cataract Refract Surg. 1991;17(5):583-91.

13. Denoyer A, Roger F, Majzoub S, Pisella PJ. [Quality of vision after cataract surgery in patients with prolate aspherical lens]. J Fr Ophtalmol. 2006;29(2):157-63. French.

14. Dietze HH, Cox MJ. Limitations of correcting spherical aberration with aspheric intraocular lenses. J Refract Surg. 2005;21(5):S541-6.

15. Kershner RM. Retinal image contrast and functional visual performance with aspheric, silicone, and acrylic intraocular lenses. Prospective evaluation. J Cataract Refract Surg. 2003;29(9):1684-94.
16. Muñoz G, Albarrán-Diego C, Montés-Micó R, RodríguezGalietero A, Alió JL. Spherical aberration and contrast sensitivity after cataract surgery with the Tecnis Z9000 intraocular lens. J Cataract Refract Surg. 2006;32(8):13207.Comment in: J Cataract Refract Surg. 2007; 33(3):359-60; author reply 360-61.

17. Chylack LT Jr, Leske MC, McCarthy D, Khu P, Kashiwagi T, Sperduto R. Lens opacities classification system II (LOCS II). Arch Ophthalmol. 1989;107(7):991-7.

18. Olsen T, Nielsen PJ. Immersion versus contact technique in the measurement of axial length by ultrasound. Acta Ophthalmol (Copenh). 1989;67(1):101-2.

19. Arshinoff SA. Dispersive-cohesive viscoelastic soft shell technique. J Cataract Refract Surg. 1999;25(2):167-73.

20. Vanden Bosch ME, Wall M. Visual acuity scored by the letterby-letter or probit methods has lower retest variability than the line assignment method. Eye. 1997; 11(Pt 3):411-7.

21. Jankov MR 2nd, Iseli HP, Bueeler M, Schor P, Seiler T, Mrochen $\mathrm{M}$. The effect of phenylephrine and cyclopentolate on objective wavefront measurements. J Refract Surg. 2006;22(5):472-81.

22. Altmann GE, Nichamin LD, Lane SS, Pepose JS. Optical performance of 3 intraocular lens designs in the presence of decentration. J Cataract Refract Surg. 2005;31(3):574-85.

23. Knorz MC, Claessens D, Schaefer RC, Seiberth V, Liesenhoff $\mathrm{H}$. Evaluation of contrast acuity and defocus curve in bifocal and monofocal intraocular lenses. J Cataract Refract Surg. 1993;19(4):513-23.

24. Kohnen T, Allen D, Boureau C, Dublineau P, Hartmann C, Mehdorn E,et al. European multicenter study of the AcrySof ReSTOR apodized diffractive intraocular lens. Ophthalmology. 2006;113(4):584. e1.

25. Souza CE, Muccioli C, Soriano ES, Chalita MR, Oliveira F, Freitas LL, et al. Visual performance of AcrySof ReSTOR apodized diffractive IOL: a prospective comparative trial. Am J Ophthalmol. 2006;141(5):827-32.

26. Leibovitch I, Lai T, Porter N, Pietris G, Newland H, Selva D. Visual outcomes with the yellow intraocular lens. Acta Ophthalmol Scand. 2006;84(1):95-9.

27. Hayashi K, Hayashi H. Visual function in patients with yellowtinted intraocular lenses compared with vision in patients with non-tinted intraocular lenses. Br J Ophthalmol. 2006; 90(8):1019-23.

28. Rawer R, Stork W, Spraul CW, Lingenfelder C. Imaging quality of intraocular lenses. J Cataract Refract Surg. 2005;31(8):1618-31.

29. Franchini A. Comparative assessment of contrast with spherical and aspherical intraocular lenses. J Cataract Refract Surg. 2006;32(8):1307-19.

30. Padmanabhan P, Yoon G, Porter J, Rao SK, Roy J, Choudhury M. Wavefront aberrations in eyes with Acrysof monofocal intraocular lenses. J Refract Surg. 2006;22(3):237-42.

31. Padmanabhan P, Rao SK, Jayasree R, Chowdhry M, Roy J. Monochromatic aberrations in eyes with different intraocular lens optic designs. J Refract Surg. 2006;22(2):172-7.

32. Rohart C, Lemarinel B, Thanh HX, Gatinel D. Ocular aberrations after cataract surgery with hydrophobic and hydrophilic acrylic intraocular lenses: comparative study. J Cataract Refract Surg. 2006;32(7):1201-5.

\section{ENDEREÇO PARA CORRESPONDÊNCIA \\ Wilson Takashi HIda}

Rua Afonso de Freitas, 488 - apto 61 - Paraíso

CEP 04006-052 - São Paulo - SP

E-mail: witaks@yahoo.com.br 